\title{
Research on the Earliest Deadline First Network Control System Dynamic Scheduling
}

\author{
Yue Wu ${ }^{\mathrm{a}}$, Linli Zhou ${ }^{\mathrm{b}}$ and Yue Duan ${ }^{\mathrm{c}}$ \\ Institute of Intelligent Machines, Chinese Academy of Sciences, Hefei 230031, China. \\ awuyue@iim.ac.cn, blinlizhou@iim.ac.cn, c13285695962@163.com
}

Keywords: Network control system, network scheduling, earliest deadline first.

\begin{abstract}
Networked control system is a subject of integrated computer technology, automatic control, communication technology and optimization theory, the effects of network scheduling on the property of network control system is a hot issue in the research of international academic circles in recent years. This paper introduces the basic composition and network control system schedulability analysis method, puts forward a network dynamic scheduling theory improved by real-time scheduling theory- Earliest Deadline First (EDF) dynamic scheduling algorithm. And the construction of multi loop networked control simulation platform based on CAN network using Truetime and Simulink toolbox. Simulation results show that, the earliest deadline first dynamic scheduling algorithm can make the performance of each loop controller in the uncertain network environment optimal.
\end{abstract}

\section{Introduction}

The Network Control System (NCS) is a system that communicates information between the nodes (sensors, controllers, and actuators) that share the communication or control the network to achieve spatial control to control the controlled object ${ }^{[1]}$. Because each node competes with the network resources, and the load capacity and bandwidth resources of the network resources are limited, the attribute of the task information in the process of information interaction is always uncertain. The nodes in the network to transmit data information and collision, there must be information transmission priorities, collision problem by introducing effective scheduling strategy to solve the information transmission network problems occur, can improve the effect on the performance of the control system of ${ }^{[2]}$.

This paper uses the CAN bus as the network environment, the earliest deadline first ([3] Earliest Deadline First EDF) scheduling algorithm to handle the task attributes of network environment and the task execution process in the uncertain problem, through the analysis of scheduling and dynamic scheduling strategy on the network, and using True Time and Matlab-Simulink simulation tools of common network system simulation control, analysis of scheduling strategy to improve the network environment, the simulation results show that the performance of EDF scheduling can effectively improve the network control system.

\section{Analysis of Dynamic Scheduling Algorithm with the Earliest Deadline}

\subsection{Overview of Network Control System Scheduling.}

NCS network scheduling and CPU real-time scheduling are similar, and there are limited shared resource constraints. At the same time, it is necessary to allocate shared resources for concurrent tasks, periodically stimulate tasks and meet the deadline requirements of ${ }^{[4]}$. In network scheduling, the competitiveness of each node to network resources corresponds to the CPU memory resources of CPU real-time task scheduling. It controls the messages that are uploaded to the network by multiple nodes in the local network and the multiple processes to be processed in the CPU processor. The dynamic scheduling theory of networked control systems is studied and the real-time scheduling theory of CPU can be used to study the scheduling problem of information during network transmission ${ }^{[3]}$. 


\subsection{Network Transmission Task Parameters.}

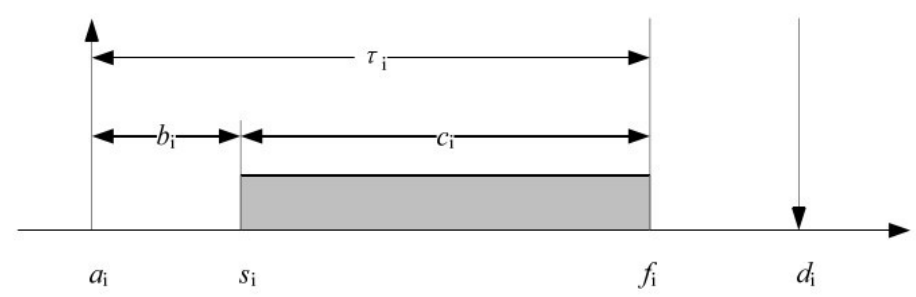

Fig. 1 Network transfer task time parameter

(1) The end of the reception $a_{i}$ : packaged information, beginning to enter the queue waiting for the queue to be sent.

(2) The starting time of the sending $s_{i}$ : the time after which the information to be sent will be packed and transmitted.

(3) The delay of competition $b_{i}$ : the time after which the messages to be sent are packed and waiting to be sent. $b_{i}=s_{i}-a_{i}$. In the control area network (CAN) transmission of data packets in the task is non preemptive and priorities, which $b_{i}$ are mainly composed of two parts: when the low priority data packet transmission for high priority data packet is sent to the waiting time, denoted by $b_{h, i}$; second is the transmission task waiting has been sent to the lower priority time required by $b_{l, i}$, so it is $b_{i}=b_{h, i}+b_{l, i}$, the maximum value is denoted as $\bar{b}_{i}, \bar{b}_{h, i}, \bar{b}_{l, i}$.

(4) Network transmission time $c_{i}$ : the time to which data packets are transmitted to the destination address via network transmission, depending on the speed of the network medium and the size of the packet.

(5) Completion time $f_{i}$ : the time point in which packets reach the destination address by network transmission.

(6) The delay of network transmission $\tau_{i}$ : the time that the information to be transmitted begins to be packaged into the transmitted information, and the time it takes for the packet to reach the destination node.

(7) Network transmission time limit (deadline) $d_{i}$ : in order to ensure the stable operation of network control system, packets containing control information are set up in the network transmission process, such as sending out timeout, response timeout and so on. These timeout times are called time frames and are generally simplified for mathematical analysis. $d_{i}=a_{i}+h_{i}$

(8) Network resource utilization $U: U=\sum_{i=1}^{n} \frac{c_{i}}{h_{i}}$, the network bandwidth is limited, and it directly affects the performance of network control system.

\subsection{EDF Dynamic Scheduling Algorithm.}

According to the real-time computer system, preemptive EDF algorithm proposed by ${ }^{[4]}$ Liu and Layland, and gives the schedulability judgment as follows: in a real-time computer system, CPU memory resource is fixed, each internal process contains different real time tasks, according to the CPU scheduling algorithm of real-time priority ordered processing each task among them, CPU; according to the dynamic allocation of task length distance deadline priority task; when the task deadline when the length of the shorter distance, the task has a higher priority; on the contrary, the longer distance. The lower priority task. The theory is extended to the research of networked control systems, and a EDF scheduling algorithm based on networked control systems is proposed. The application research shows that the algorithm is the best dynamic priority scheduling algorithm.

In the network control system, $\mathrm{n}$ non-preemptive periodic sampling tasks ( $\mathrm{i}=1$ indicates the highest priority according to the decreasing priority of the task, $\mathrm{i}=\mathrm{N}$ represents the lowest priority), if for all $i=1,2$, 3 meet: 


$$
\frac{c_{1}}{h_{1}}+\frac{c_{2}}{h_{2}} \cdots \frac{c_{i}}{h_{i}}+\frac{\bar{c}_{l, i}}{h_{i}} \leq 1
$$

In the formula, $\bar{b}_{l, i}$ is the longest time that the i-th transmission task is blocked with the priority of the task, that is, $\bar{b}_{l, i}=\max c_{j}, j=i+1, \cdots N$, the task based on the EDF algorithm is schedulable in the network control system.

\subsection{Dispatch Analysis.}

Non-preemptive priority EDF algorithm can be dispatched theorem analysis: Let $T=\left\{T_{1}, T_{2}, \cdots T_{n}\right\}$ there is a transmission task, in which a series of the task set $T_{i}\left(c_{i}, h_{i}\right)$ in ascending order of the order, $c_{i}$ for the network transmission task transmission time, that is, transmission delay, $d_{i}$ is the deadline $\left(d_{i}=h_{i}\right.$ ) of the transmission task $T_{i}$, the set of tasks can be scheduled in the non-preemptive priority EDF scheduling algorithm if and only if the following conditions are true:

$$
\begin{aligned}
& U=\sum_{i=1}^{n} \frac{c_{i}}{h_{i}} \leq 1 \\
& \forall i, 1 \leq i \leq n ; h_{1} \leq t \leq h_{i} ; t \geq c_{i}+\sum_{j=1}^{i-1}\left[\frac{t-t^{\prime}}{h_{j}}\right] c_{j}, t \leq c_{i}
\end{aligned}
$$

There are four loops for the multi-loop network control system. The time attribute (unit: ms) of the transmission task is as follows:

$$
\begin{array}{llll}
i=1 & d_{i}=4 & h_{i}=4 & c_{i}=1 \\
i=2 & d_{i}=6 & h_{i}=6 & c_{i}=2 \\
i=3 & d_{i}=8 & h_{i}=8 & c_{i}=2 \\
i=4 & d_{i}=16 & h_{i}=16 & c_{i}=2
\end{array}
$$

Let $t=h_{i}-1, t^{\prime}=1$, the network utilization rate is:

$$
U=\sum_{i=4}^{4} \frac{c_{i}}{h_{i}}=\frac{1}{4}+\frac{2}{6}+\frac{2}{8}+\frac{2}{16}=0.958<1
$$

The following lists the time-dependent relationship of the task set when i takes different values:

$$
\begin{aligned}
& i=2, t=5 ; c_{2}+\sum_{j=1}^{1}\left[\frac{t-t^{\prime}}{h_{j}}\right] * c_{j}=2 \\
& i=3, t=7 ; c_{3}+\sum_{j=1}^{2}\left[\frac{t-t^{\prime}}{h_{j}}\right] * c_{j}=5 \\
& i=4, t=15 ; c_{4}+\sum_{j=1}^{3}\left[\frac{t-t^{\prime}}{h_{j}}\right] * c_{j}=11
\end{aligned}
$$

When i take different values, can meet $t \geq c_{i}+\sum_{j=1}^{i-1}\left[\frac{t-t^{\prime}}{h_{j}}\right] c_{j}$. Therefore, the theorem we can see that the task set can be scheduled.

\section{Experimental Simulation and Result Analysis}

\subsection{Construction of Network Control System Simulation Platform.}

A network control system simulation toolbox developed by The Lund University Institute of Technology and Anton Cervin and Dan Henriksson and other scholars: Truetime, which can support real-time simulation control and network scheduling, followed by a simulation of network 
transmission delay, network parameters on the performance of the control system of other aspects, so as to provide a basis for the simulation of networked control system.

Simulink and Truetime toolbox are used to build a three control loop network control system simulation platform. Simulation model shown in Fig. 2, three identical DC motor model and the corresponding PID controller in the same CAN network environment. The controller, the actuator and the scheduler adopt the event-driven mode, in which the dispatcher is driven by the internal three-stage sampling module, and the sampling period is $h_{1}=2 m s, h_{2}=5 m s, h_{3}=7 m s$.

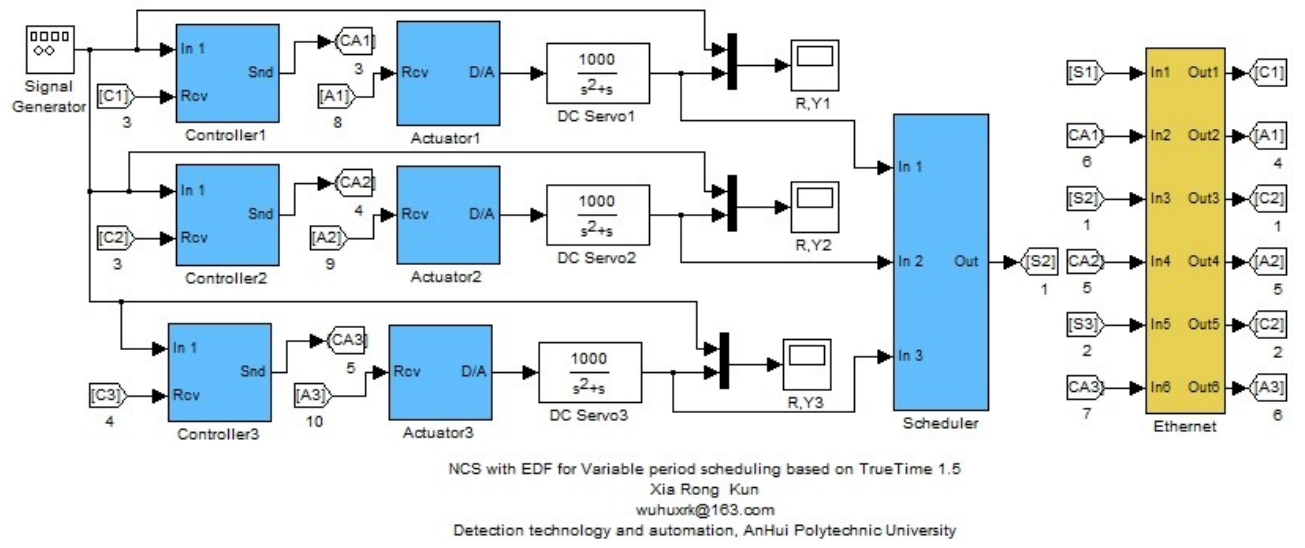

Fig. 2 Simulation model of networked control system

\subsection{Simulation Results.}

EDF dynamic scheduling policy, interference nodes and scheduler, controller and other nodes scheduling, as shown in figure 3. The dynamic interference nodes occupy the network bandwidth, the highest priority, the scheduler node and controller node and actuator nodes are not idle on the network to send data, but according to different data transmission control loop length distance deadline to send priority on their dynamic adjustment.

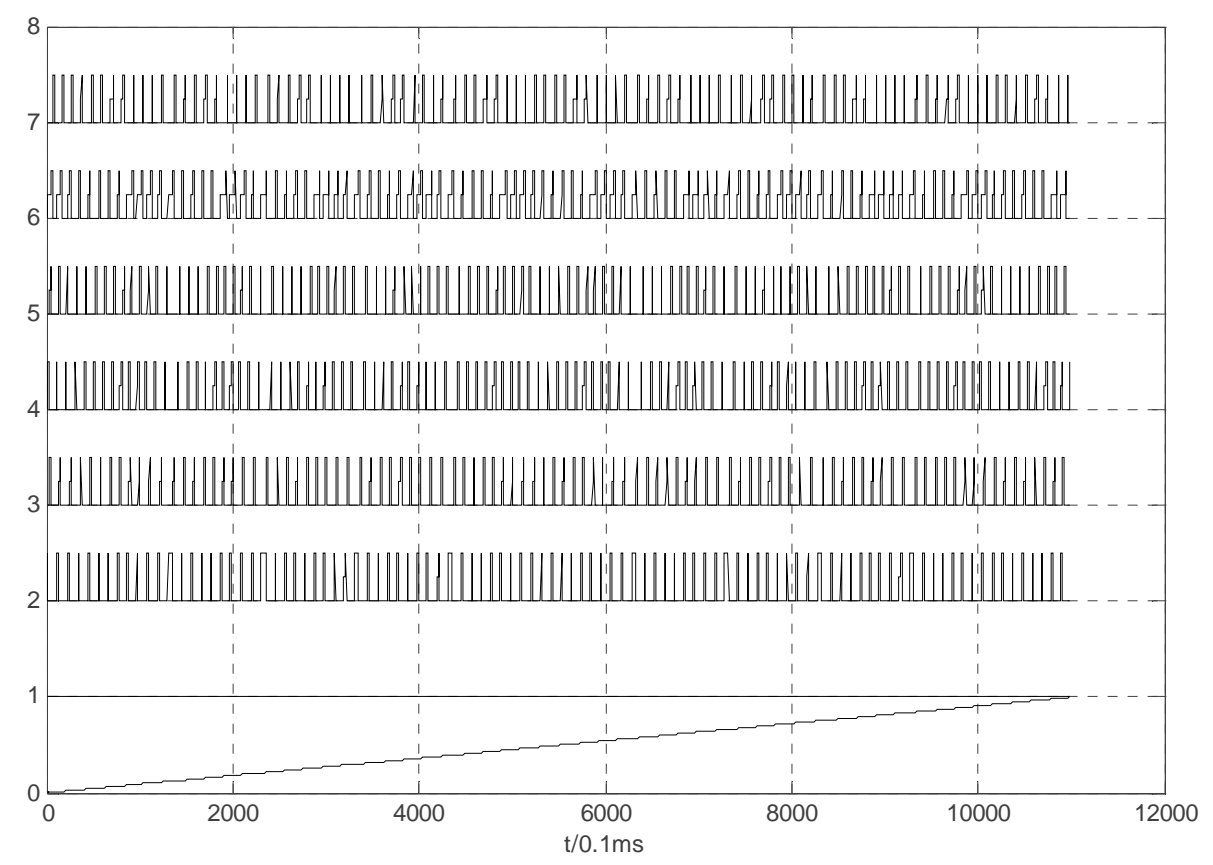

Fig. 3 Three loop EDF policy scheduling diagram

In Fig. 3, the spike in the data transfer sequence diagram of the sampling module in the scheduler is due to the contention that the node and the higher priority node simultaneously contend for network transmission rights. 

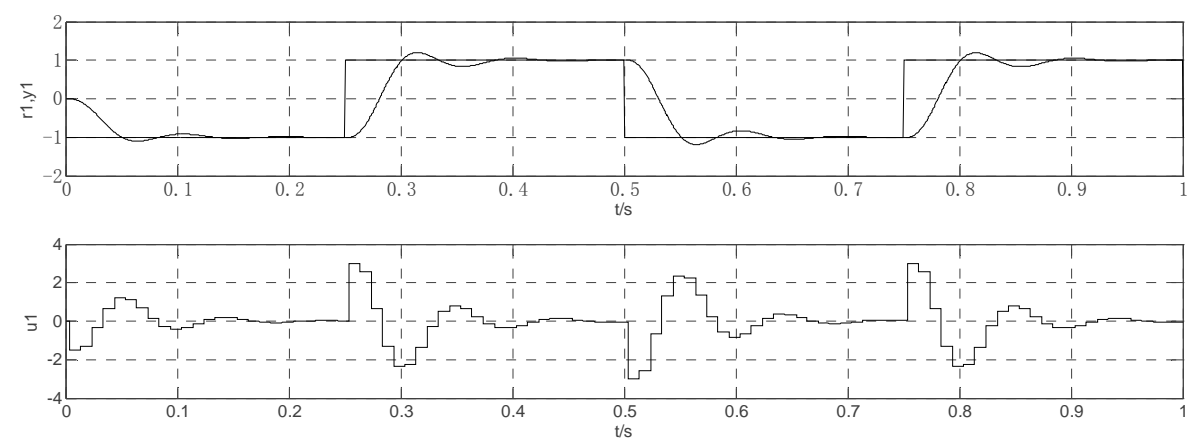

Fig. 4 Loop 1 square wave response and control rate
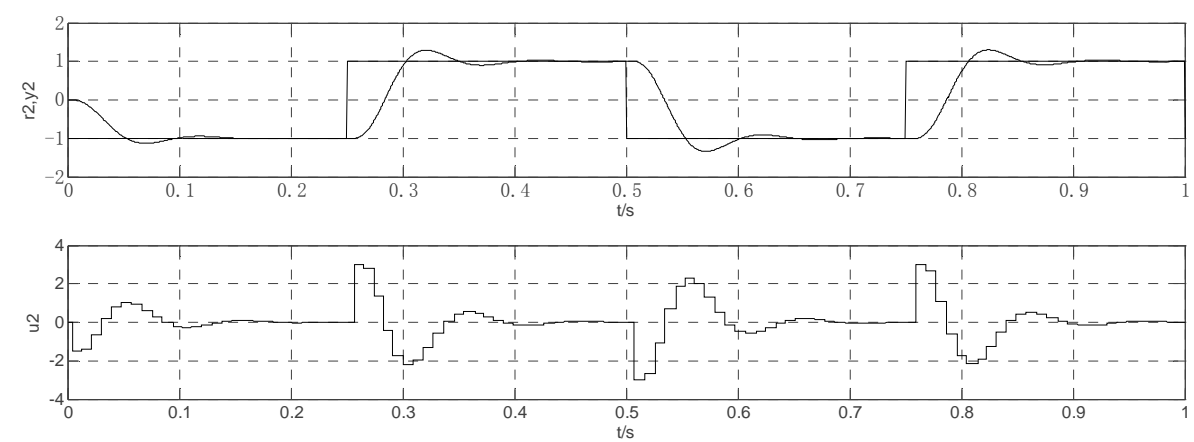

Fig. 5 Loop 2 square wave response and control rate
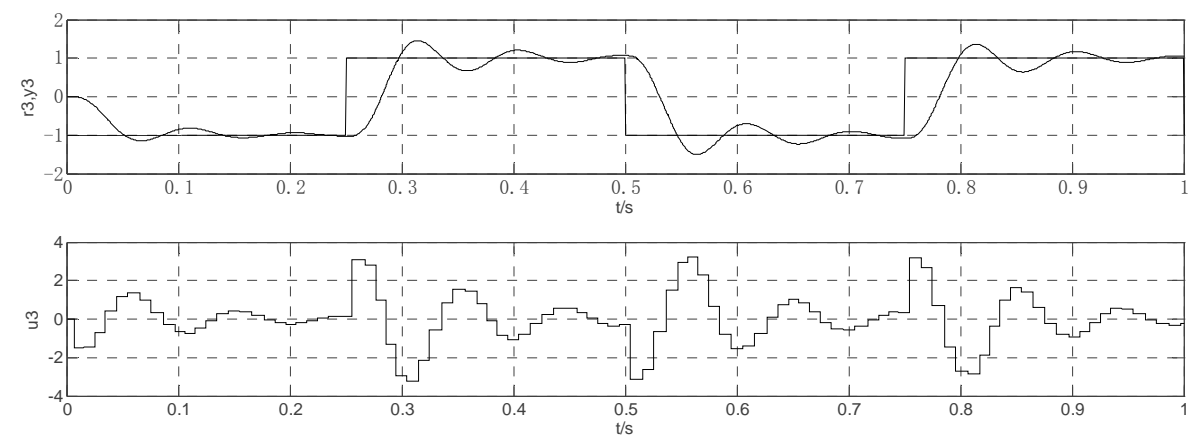

Fig. 6 Loop 3 square wave response and control rate

Three control loops in the network control system of square wave response and the corresponding control rate as shown in Fig. 4, Fig. 5, and Fig. 6, it can be seen from the figure, the cyber source is limited and unstable, EDF Dynamic Scheduling Considering the urgency of different loop data, so it can make the system performance have been greatly the improvement.

\section{Conclusion}

Network scheduling affects the performance of networked control systems or to a great extent, which is under the environment of network, the nodes of multiple control loops in the common occupation of limited cyber source distribution, cyber source reasonable and how to determine the priority of data packets is the main problem facing network control system. In this paper, the real-time scheduling theory is applied to the network control system, which leads to an optimal dynamic priority scheduling algorithm - the earliest deadline-priority dynamic scheduling algorithm. The algorithm can be dynamically allocated according to the length of the scheduled task deadline Task priority, with strong environmental adaptability. The simulation results show that the algorithm can improve the stability of the network control system in the network control system. 


\section{Acknowledgements}

This work was supported by the National Science \& Technology Pillar Program during the 12th Five-year Plan Period (Grant No. 2015BAD18B05).

\section{References}

[1]. Antsaklis P, Baillieul J. Special issue on technology of networked control systems[C]. Proceedings of IEEE Special Issue on the Emerging Technology of Networked Control Systens,2007,95(1):5 8.

[2]. Jeffek K,Stanat D F,Martel C U.On non-preemptive scheduling of periodic and sporadic tasks[C]//Proc of the 12th Real-time System Symposium. Washington DC:IEEE Computer Socity,1991:129-139.

[3]. Velasco M.A control approach to bandwidth management in networked control systems [C]:Proceedings of the 30th IEEE Annual Conference on Industrial Electronics Society,2004:2343 2348

[4]. Liu C L,Layland J W.Scheduling algorithms for multiprogramming in a hard real-time environment[J].Journal of the ACM,1973,20(1):46-61. 